\title{
Adaptive changes between cue abstraction and exemplar memory in a multiple-cue judgment task with continuous cues
}

\author{
LINNEA KARLSSON \\ Umeå University, Umeå, Sweden \\ Peter Juslin \\ Uppsala University, Uppsala, Sweden \\ AND \\ HenRIK OlsSON \\ Uppsala University, Uppsala, Sweden \\ and Max Planck Institute for Human Development, Berlin, Germany
}

\begin{abstract}
The majority of previous studies on multiple-cue judgment with continuous cues have involved comparisons between judgments and multiple linear regression models that integrated cues into a judgment. The authors present an experiment indicating that in a judgment task with additive combination of multiple continuous cues, people indeed displayed abstract knowledge of the cue criterion relations that was mentally integrated into a judgment, but in a task with multiplicative combination of continuous cues, people instead relied on retrieval of memory traces of similar judgment cases (exemplars). These results suggest that people may adopt qualitatively distinct forms of knowledge, depending on the structure of a multiple-cue judgment task. The authors discuss implications for theories of multiple-cue judgment.
\end{abstract}

Judgments that demand integration of information occur repeatedly throughout our lives. One professional example is that of a stock broker who considers multiple economic indices to make a judgment of the future interest rate. From the outset, the study of multiple-cue judgments that involve multiple continuous cues (e.g., economic indices) and a continuous criterion (e.g., interest rate) has been concerned with the statistical modeling of judgment data (see Cooksey, 1996; Hammond \& Stewart, 2001). Multiple-cue judgment often is well fitted by linear models, based on few cues that are used inconsistently, and with poor subjective insight into the cue weights (Cooksey, 1996). Although many researchers have emphasized that these models summarize the output of the judgment process, rather than the process itself (e.g., Hoffman, 1960), the more or less explicit interpretation in the literature of multiple-cue judgment has often been that of controlled mental integration of the cues to make a judgment (e.g., Einhorn, Kleinmutz, \& Kleinmutz, 1979; Juslin, Olsson, \& Olsson, 2003). Although studies have investigated cognitive process models in single-cue probability learning (e.g., DeLosh, Busemeyer, \& McDaniel, 1997; Kalish, Lewandowsky, \& Kruschke, 2004) and multiple-cue judgment with binary cues (e.g., Juslin, Karlsson, \& Olsson, in press; Juslin et al., 2003; Olsson, Enkvist, \& Juslin, 2006; Olsson, Juslin, \& Olsson, 2006), to our knowledge few at- tempts have been made to model the cognitive process in multiple-cue judgment with continuous cues.

We attempt to identify the cognitive processes behind multiple-cue judgment with continuous cues by cognitive modeling. According to Shiffrin and Schneider's (1977) classical distinction between automatic and controlled processing, controlled processes are serial and require attention. Drawing on this knowledge of controlled cognitive processing, we predict and verify task conditions in which people mentally integrate knowledge of individual cue-criterion relations and task conditions in which they rely on memory for similar judgment cases (exemplars). This change in the representation has been verified with binary cues (Juslin et al., in press), but, because continuous cues may be inherently more conducive to abstracting the individual cue-criterion relations and may render more cue combinations (i.e., exemplars) in these tasks, the existence of such adaptations is still an open question.

Juslin et al. (in press) proposed that multiple-cue judgment is serial and capacity-constrained, and therefore tends to involve consideration of two successive criterion values. The criterion values of two successive exemplars with different values on a cue may be compared in order to infer the effect of the cue on the criterion. For example, a large difference in blood pressure (the criterion) for two patients similar

L. Karlsson, karlsson@mpib-berlin.mpg.de 
in regard to all cues but one, body weight, may suggest that this difference predicts the difference in blood pressure.

At the time of judgment, the constraint to consider two criterion values implies a sequential adjustment, whereby one estimate of the criterion is updated into another estimate (Anderson, 1981; Hogarth \& Einhorn, 1992). A physician may make one estimate of a patient's blood pressure prior to considering the patient's body weight, which is updated after taking body weight into account. Juslin et al. (in press) illustrated that this sequential process is compatible both with additive integration of cues (cue abstraction), as when symptoms are considered in sequence, and with additive integration of exemplars, as when diseases of similar patients are considered (exemplar memory; see Medin \& Schaffer, 1978).

When this capacity-constrained process involves cue abstraction, two implications are important to our argument: (1) The cue-criterion knowledge becomes biased to represent linear relations. The constraint mainly to consider two sets of cue-criterion values in training allows detection of linear relations, but nonlinear relations demand simultaneous consideration of at least three cue-criterion pairs. As an analogue, in a bivariate plot with only two pairs of $x, y$ values, it is impossible to detect a nonlinear relationship between the two variables. (2) Iterative adjustment implements additive integration of the effect of cues on the criterion, whereby the impact of the new cue is added to the previous estimate.

Cue abstraction is biased to represent linear additive functions between cues and criterion. There is no doubt that there exist tasks for which linear additive cue integration is a poor approximation - for example, when judging the level of voltage in a cable as a function of Ohm's law or motion according to Newton's laws. We should therefore be equipped also with means to learn in nonlinear and nonadditive tasks. One hypothesis is that the judge adopts another representation and relies on exemplar memory (Juslin et al., in press). This hypothesis predicts that, despite otherwise identical stimuli and procedures, a multiple-cue judgment task in which the cue combination is additive will invite cue abstraction, but a task in which the rule is multiplicative will enforce adoption of exemplar memory.

\section{The Experiment}

To test this prediction, we manipulated whether the combination rule in a multiple-cue judgment task was additive or multiplicative. We also manipulated the cuecriterion function to be either positively related to the criterion for all four cues (homogeneous condition) or positively related to the criterion for two cues and negatively related for the other two (heterogeneous condition). The cover story concerns the effectiveness of species of herbs as medical treatments for a virus. The effectiveness is measured as the maximal amount of a chemical substance (in milligrams) that can be extracted from the species. The species have four continuous dimensions $\left(C_{1}, C_{2}, C_{3}, C_{4}\right)$, and each dimension varies between 0 and 10 .

In the additive task, the criterion $c$ is a linear, additive function of the cues:

$$
c=500+4 C_{1}+3 C_{2}+2 C_{3}+1 C_{4}+\varepsilon .
$$

$C_{1}$ is the most important cue, with a coefficient of 4 (i.e., a relative weight of .4); $C_{2}$ is the second most important, with a coefficient of 3 ; and so forth. The cues are uncorrelated. $\varepsilon$ is a normally and independently distributed random error with a standard deviation that produces a multiple correlation $R$ between cues and criterion of .9 (i.e., the ecological validity of the cues).

In the multiplicative task, the criterion $c$ is a multiplicative function of the four cues,

$$
c=509.05+0.54 \cdot e^{\left(4 C_{1}+3 C_{2}+2 C_{3}+1 C_{4}\right) / 18}+\varepsilon,
$$

with the same coefficients $(4,3,2$, and 1$)$ as in the additive task (Equation 1). To distinguish between cue abstraction and exemplar memory, there was a training phase with criteria between 510 and 590 for both tasks, and a test phase containing both previously seen old exemplars and new exemplars not previously encountered in training, including exemplars outside the training range. The criterion range was the same in both conditions during training, but the different integration rules naturally implied different test ranges (500-600 in the additive task, and 509-650 in the multiplicative task).

Figure 1 illustrates quantitative model predictions (see the Appendix) for 44 judgment cases in the test phase (the scatter in the graphs is because of the probabilistic criterion). The additive cue-abstraction model implies adding up the impact of individual cues. Although such a model is prohibited by the description of controlled judgment given above (Juslin et al., in press), for completeness we also considered a multiplicative cue-abstraction model (Equation 2 with subjective weights). With both models, if the weights are learned roughly correctly in training, cue abstraction predicts that the most extreme judgments will be made for the extreme new exemplars in the test phase with criterion values outside the training range, even if they have never been encountered in the training phase. Depending on the magnitude of the cue weights, these judgments can extrapolate beyond the training range (510-590).

The exemplar model involves a weighted average of the criteria observed in training, with the most similar exemplars receiving the largest weight (see the Appendix). Therefore, it cannot extrapolate beyond the training range and does not make the most extreme judgment for the new extreme test exemplars (DeLosh et al., 1997). In contrast to the cue-abstraction models, in which old and new exemplars obey the same function, the exemplar model predicts different data patterns for old and new exemplars, since old exemplars benefit from the possibility of retrieving an exactly matching exemplar from memory, but new exemplars do not.

We examined (1) judgment performance for the old and new judgment cases, in which cue abstraction suggests no old-new differences but exemplar memory predicts large old-new differences; (2) the proportion of extrapolation judgments outside of the training range (510-590), for which the existence of such extrapolation for new exemplars is consistent with cue abstraction but inconsistent with exemplar memory; and (3) the fit of the models examining the detailed quantitative agreement between data and model predictions. 
A

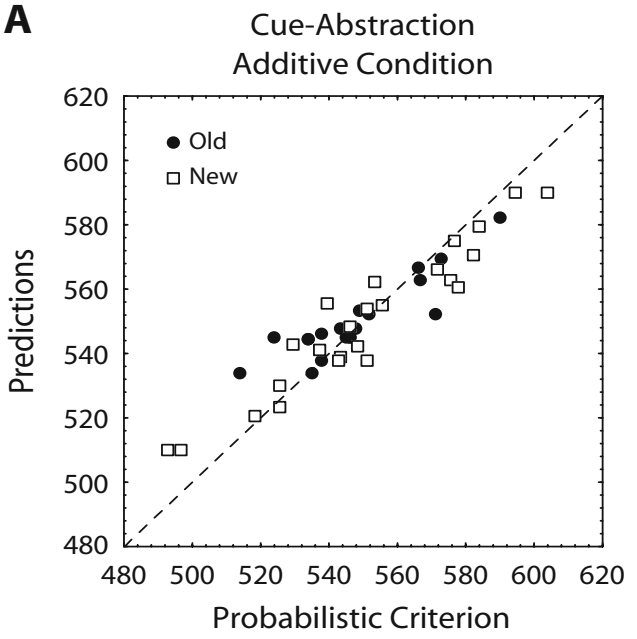

C

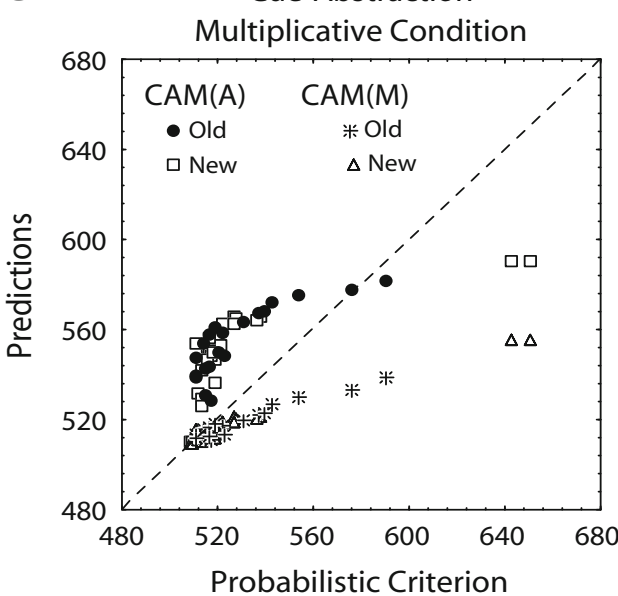

B
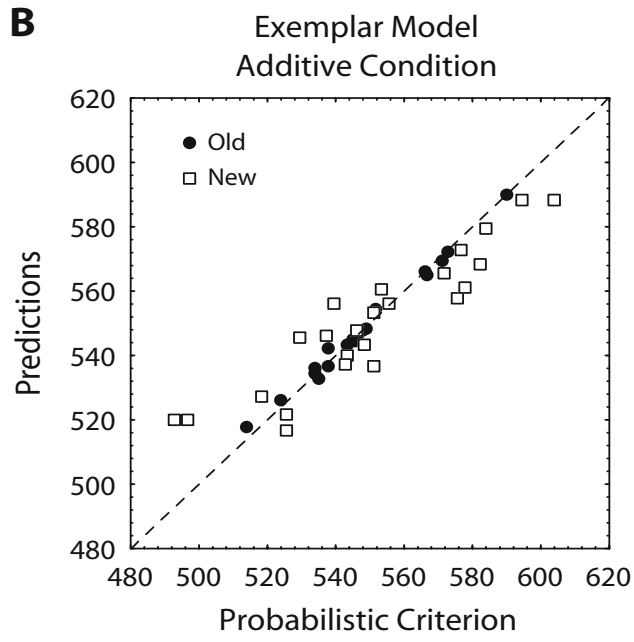

D

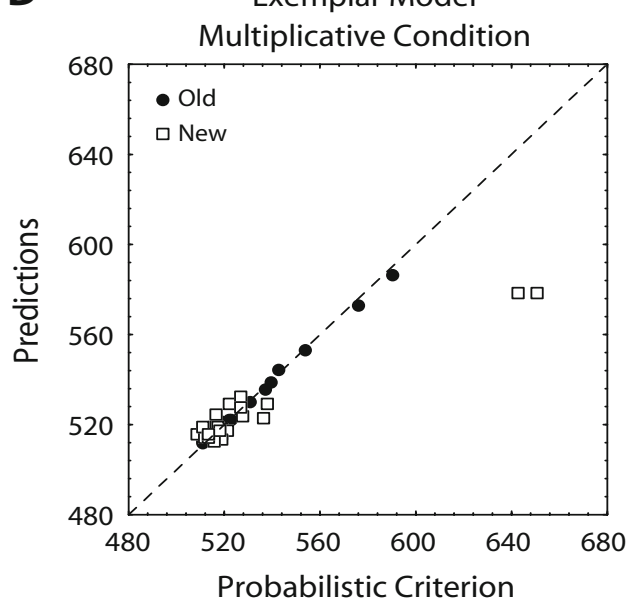

Figure 1. Predictions by cue-abstraction model (CAM) and exemplar-based model (EBM) in additive and multiplicative task environments. (A) CAM in the additive task environment (with slightly regressive weights: 3.2, 2.4, 1.6, and 0.8). (B) EBM in the additive task environment $(s=.25$ and $h=4)$. (C) Additive [CAM(A)] and multiplicative [CAM(M)] cue-abstraction models in the multiplicative task environment (with weights 3.2, 2.4, 1.6, and 0.8). (D) EBM in the multiplicative task environment $(s=.25$ and $h=4)$. The choice of values for the parameters is arbitrary and only used for illustrative purposes. A similar pattern for EBM results if the ss are chosen to correspond to the "true" weights. The identity lines in the graphs represent perfectly accurate judgments. The probabilistic criterion values on the $x$-axis are not computed anew for old exemplars.

\section{METHOD}

\section{Participants}

Thirty-two undergraduate students volunteered and were paid 60-99 Swedish kronor, depending on performance. Twenty were male and 12 were female, all between the ages of 20 and 32 .

\section{Design, Materials, and Procedure}

A $2 \times 2$ factorial between-subjects design was used, with 16 participants in the additive and 16 in the multiplicative version of the task. Eight participants in each task did the homogeneous version, and 8 did the heterogeneous version. In the training phase, the participant learned to judge the effectiveness of species of an herb from outcome feedback, given with two decimals. The species were shown as four written propositions on a computer screen. At each trial, the participant answered the question "How many milligrams of Ranulin does this species contain?" The four dimensions were weeks in bloom, optimal amount of iron in the ground, degrees of latitude at which the plant does well, and amount of water it emits per leaf area. Each dimension varied in 11 equidistant steps $(0-10)$. A random sample of 300 exemplars for each participant was drawn from among the $11^{4}$ possible exemplars, allowing only those exemplars with criterion values between 510 and 590. A 2-min pause was given after 150 trials.

In the test phase, participants judged the species effectiveness, but without outcome feedback. The test phase consisted of 44 judgments: (1) 20 randomly chosen old exemplars shown in training; (2) 20 randomly chosen new exemplars drawn from the training distribution; and (3) 4 extreme exemplars with criterion values outside the training range (e.g., with cue values $[0,0,0,0]$ and $[10,10,10$, $10])$. In the condition with heterogeneous cue directions, for half of the participants the negative sign was assigned to cues with weights 4 and 2 , and for the other half to the cues with weights 3 and 1 .

\section{Dependent Measures}

Judgment accuracy is measured by root-mean square error (RMSE) between judgment and criterion. Extrapolation is measured 
by the proportion of judgments at test falling outside of the training range (510-590), as computed separately for old and new exemplars for each participant. Extrapolation for both old and new is not predicted by the cue-abstraction model, but could arise if the judgments are extremely erratic, leading a number of judgments to fall outside of the training range by chance, or if the cue weights are erroneously learned. Given reliance on cue abstraction in the additive task and exemplar memory in the multiplicative task, the crucial prediction is of extrapolation only for new extreme exemplars in the additive task. Model fit is indexed by root-mean square deviation (RMSD) between predictions and judgments in the test phase.

\section{RESULTS}

\section{Performance at Training}

To establish that a difference at test is not due to unequal performance at the end of training, training data were analyzed in six blocks, with 50 judgments in each block. In the last block, there was no difference in performance in terms of RMSE between the additive and multiplicative tasks $[M=16.0$ vs. 13.4 , respectively; $F(1,27)=2.604$, $\left.M S_{\mathrm{e}}=52.5, p=.118\right]$. The participants in the homogeneous condition, however, performed somewhat better than those in the heterogeneous condition $[M=12.7$ vs. 16.7, respectively; $\left.F(1,27)=5.935, M S_{\mathrm{e}}=119.6, p<.05\right]$. The interaction was not significant.

\section{Mean Judgments at Test}

In Figure 2, mean judgments for each test exemplar are plotted against the criterion. In the additive task (Figures $2 \mathrm{~A}$ and $2 \mathrm{C}$ ), the judgments are a linear function of the criterion, with the most extreme judgments for the new extreme exemplars. As predicted by cue abstraction (Figure 1A), which suggests no systematic differences between old and new exemplars, the best-fitting regression lines for old and new judgments coincide. In the multiplicative task (Figures $2 \mathrm{~B}$ and $2 \mathrm{D}$ ), the judgments for new exemplars clearly deviate both from the identity line (perfect judgment) and the best-fitting regression line for old exemplars. As predicted by the exemplar model (Figure 1D), here there is a systematic difference between the old and new exemplars. Although the judgments are a positive function of the criteria in the training range, the judgments are not extrapolated beyond the training range. The pattern is basically the same in both cue direction conditions, except that judgment performance is generally poorer in the heterogeneous cue conditions, with more scatter in Figures 2C and 2D.

\section{Performance at Test}

An ANOVA on judgment error RMSE with task and cue direction as between-subjects variables and old versus new exemplar as a within-subjects variable showed three significant main effects and two interactions, in which the interactions are more important for the interpretation. There was a significant interaction between task and old versus new exemplar $\left[F(1,28)=121.85, M S_{\mathrm{e}}=1,098, p<.001\right]$. For old exemplars, learning was similar in the tasks, but for the new exemplars, performance was especially poor in the multiplicative task. This is the interaction predicted if cue abstraction dominates in the additive task, producing little or no systematic difference between old and new exemplars, but exemplar memory becomes more important in the multiplicative task, implying worse performance for new exemplars. There was also an interaction between cue direction and old versus new exemplar $\left[F(1,28)=6.23, M S_{\mathrm{e}}=56, p<.05\right]$. For old exemplars, performance was similar in the homogeneous and heterogeneous conditions. For new exemplars, performance was poorer than for old exemplars, especially in the heterogeneous condition. This suggests that exemplar memory became more prevalent in the heterogeneous condition, in which the cue-criterion structure is more complex.

The significant main effects - better performance in the additive task $\left[F(1,28)=11.7, M S_{\mathrm{e}}=687, p<.01\right]$, with homogeneous cue directions $\left[F(1,28)=7.63, M S_{\mathrm{e}}=448\right.$, $p<.05]$, and with old exemplars $\left[F(1,28)=167.93, M S_{\mathrm{e}}=\right.$ $1,513, p<.001]$ - are mainly secondary to these significant interactions. Because there were no significant interactions between task and cue direction and the cell sizes were relatively small $(n=8)$, we concentrate on the main effects of task and cue direction in the ensuing analysis.

\section{Extrapolation}

The median proportion of extrapolation judgments outside the interval 510-590 is 0 for both old and new exemplars in the multiplicative task and for old exemplars in the additive task (interquartile index of 0 in all three cases). The median proportion of extrapolation judgments is .087 (interquartile index .166) for new exemplars in the additive task. This distribution is significantly different from the distributions in the three other cells, in all cases with $p<.005$ by nonparametric statistical tests. There are no significant differences between the old and new exemplars in the homo- and heterogeneous conditions: The medians there fall between 0 and .025 in all four cells. The results thus indicate extrapolation only for new exemplars in the additive task. This is the pattern expected if cue abstraction dominates in the additive task and exemplar memory dominates in the multiplicative task. No corresponding patterns were observed as a function of the manipulation of the cue directions.

\section{Quantitative Model Fits}

Entering task (between subjects), cue direction (between subjects), and model (within subjects) as independent variables in an ANOVA, with model fit RMSD as the dependent variable, yields a significant main effect of model $[F(1,21)=$ $\left.5.08, M S_{\mathrm{e}}=7.41, p<.05\right]$ and a significant interaction $\left[F(1,21)=7.40, M S_{\mathrm{e}}=10.80, p<.05\right]$. The interaction is the key effect, implying that the additive cue-abstraction model is better in the additive task $\left(\mathrm{RMSD}_{\mathrm{CAM}}=10.13 \mathrm{vs}\right.$. $\mathrm{RMSD}_{\mathrm{EBM}}=11.85$ ), but this superiority disappears in the multiplicative task, in which the exemplar model shows a better fit $\left(\mathrm{RMSD}_{\mathrm{EBM}}=9.18 \mathrm{vs}\right.$. $\left.\mathrm{RMSD}_{\mathrm{CAM}}=9.39\right)$. Looking at the proportion of participants best fitted by cue abstraction or by exemplar memory, in the additive task the cue-abstraction model indicated better fit than the exemplar model for $83 \%$, whereas the exemplar model provided a better fit for $17 \%$. In the multiplicative task, the exemplar model indicated better fit than the cue-abstraction model for $62 \%$, whereas the cue-abstraction model provided a better fit for $38 \%$. This difference in the proportions is significant $\left[\chi^{2}(1)=5.24, n=25, p<.05\right]$. The multiplicative cue- 
A

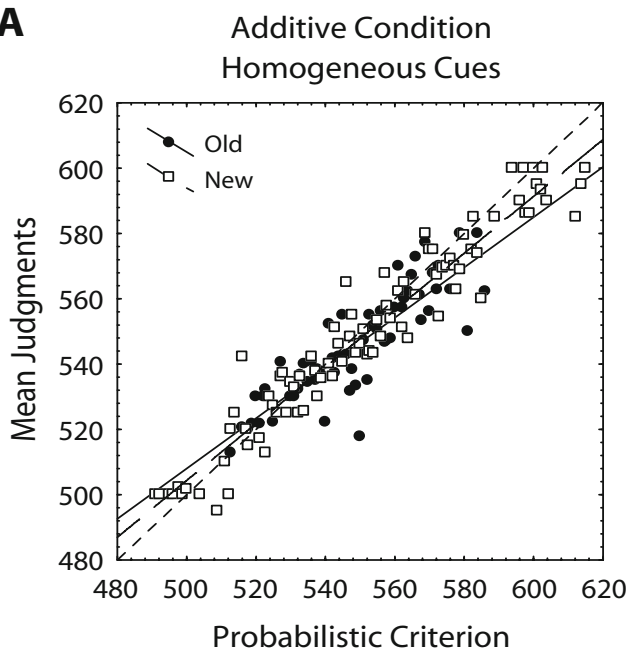

\section{C

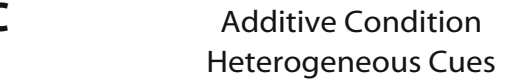

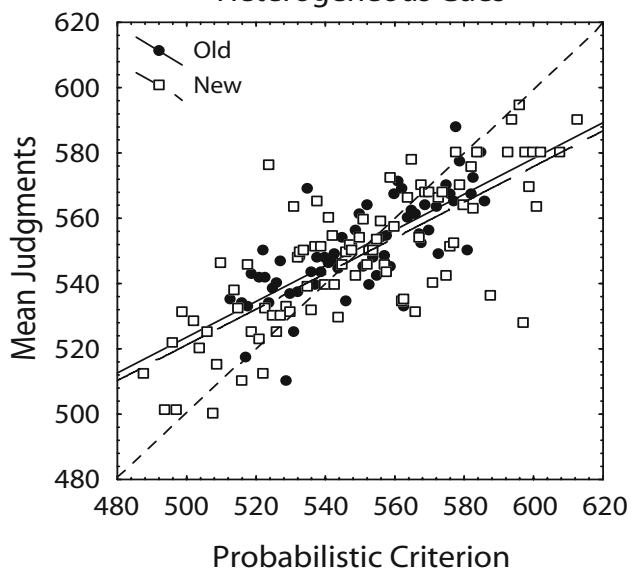

B
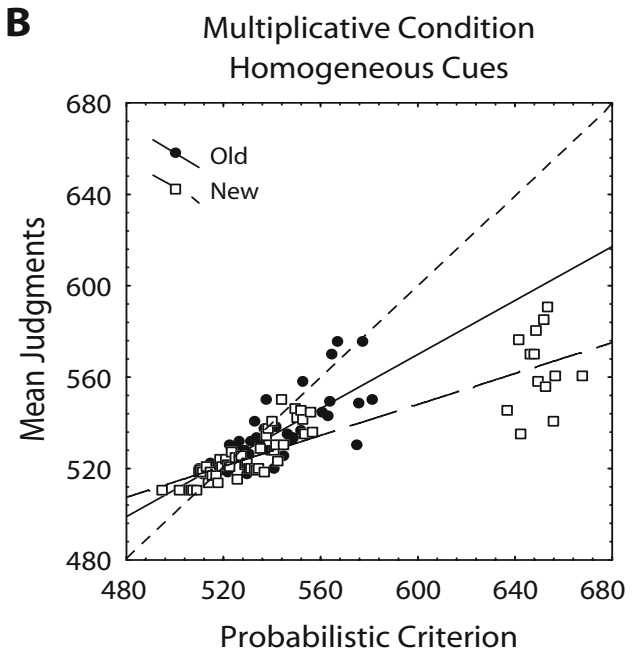

D
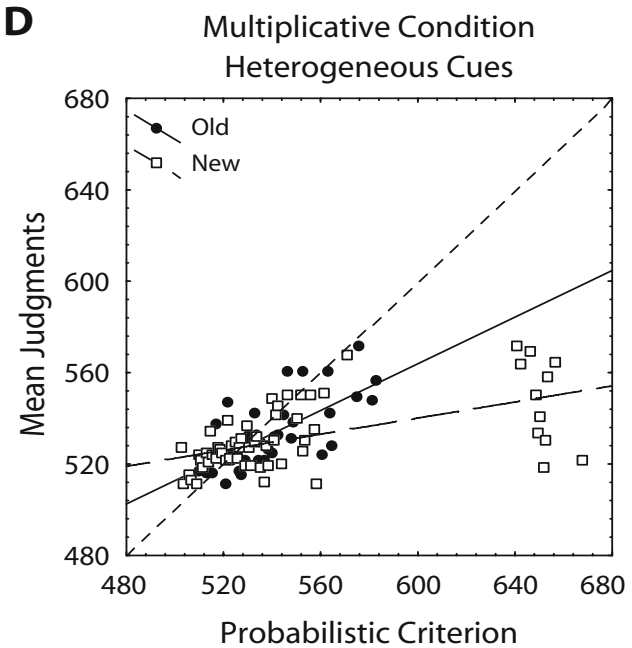

Figure 2. Mean judgments for the different tasks and conditions: (A) additive, homogeneous; (B) multiplicative, homogeneous; (C) additive, heterogeneous; (D) multiplicative, heterogeneous. Best-fitting regression lines are based on either the old exemplars seen in training (solid lines) or the new exemplars introduced at test (broken lines). The identity lines in the graphs (dotted lines) represent perfectly accurate judgments. The probabilistic criterion values on the $x$-axis are not computed anew for old exemplars.

abstraction model is the poorest model in the multiplicative task, although it provides the best account for $23 \%$ of the participants (62\% were best accounted for by the exemplar model). This evidence for multiplicative cue abstraction for $23 \%$ is spurious, however, because there are no signs of extrapolation and there are different linear slopes for the new and old exemplars. There was no corresponding difference in the proportions best accounted for by each model as a function of cue directions $(p=.624)$. The model fits thus reinforce the idea that participants mainly rely on cue abstraction in the additive task, whereas in the multiplicative task they mainly rely on exemplar memory.

\section{A Replication With Equally High Extreme Values at Test}

The training criterion range was the same in both tasks to ascertain that the combination rule was the only difference in the training phase, where the cognitive processes are formed. By necessity, however, when unconstrained the function forms yield different extreme values at test. Is the difference between the two tasks explained by this difference in the test criterion range? A new data collection with an otherwise identical multiplicative task $(N=16$; $n=8$ homogeneous, $n=8$ heterogeneous) was therefore made, in which the high-extreme value in the test phase was constrained to be 600, exactly as in the additive task.

Comparing this replication with the original experiment (Figure 3) yields the conclusion that, even though performance on new exemplars in terms of RMSE is a bit better in this replication than with the original extreme values in the multiplicative task, it is still significantly poorer than the judgments for new exemplars in the additive task. The important interaction between additive (original experiment) versus multiplicative task and old versus new exemplar is also significant for the replication data $\left[F(1,28)=29.59, M S_{\mathrm{e}}=218.8, p<.001\right]$. 
In regard to extrapolation, for the high-extreme exemplars, only one single response is outside of the training range. Thus, for the measures of performance and extrapolation, the new data set replicates the data pattern from the original data collection; that is, worse performance on new than on old exemplars and no extrapolation beyond the training range. In regard to the model fit, however, there was no significant difference between the models. This is not too surprising, given that the replication deliberately excluded the high-extreme test exemplar most diagnostic for distinguishing between the two models (see Figure 1).

\section{DISCUSSION}

Consistently across the analyses, the results support the hypothesis of an adaptive change in the representations that underlie multiple-cue judgments in these tasks with continuous cues, as a consequence of the structural properties of the task. The different analyses complement and reinforce each other. The performance measures indicate no significant old-new differences in the additive task, but substantial oldnew differences in the multiplicative task. The extrapolation measure in the additive task is consistent with cue abstraction but inconsistent with exemplar memory. Finally, there is a significant interaction in regard to quantitative model fit, with better fit of cue abstraction in the additive task and better fit of exemplar memory in the multiplicative task.

The additive and multiplicative tasks involve exactly the same stimuli, except for the criterion that is either generated by an additive or a multiplicative cue combination rule. Yet, when the combination rule is additive, the judgment process appears to conform to mental integration of abstracted knowledge of cue-criterion relations, but with a nonadditive cue combination rule, exemplar memory is the dominating process. The results indicate that this adaptive change may also pertain in tasks with continuous cues.

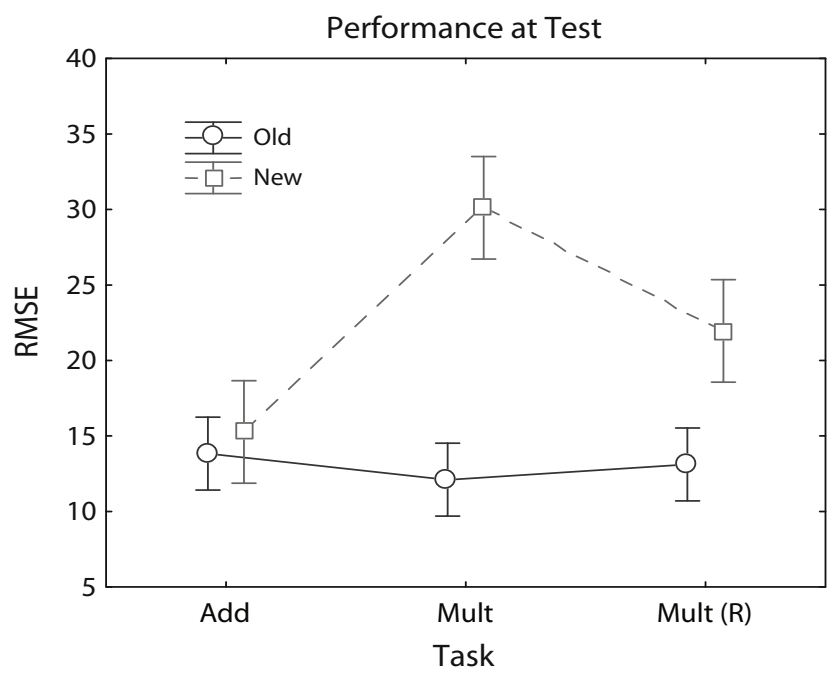

Figure 3. Judgment error in terms of mean root-mean square error (RMSE), with $95 \%$ confidence intervals, for old and new exemplars in the test phases. "Add" refers to the additive task, "Mult" to the multiplicative task, and "Mult (R)" to the replication of the multiplicative task.
These results support the hypothesis that the sequential, capacity-constrained nature of controlled judgment processes hinders mental integration of abstracted knowledge of the effect of cues on the criterion when the combination rule is nonadditive (Juslin et al., in press). There is evidence for a rule bias in categorization learning (Ashby et al., 1998), and a similar initial preference for abstract knowledge is also likely in multiple-cue judgment tasks. Some participants in the multiplicative task may have been caught in persistent but futile attempts at cue abstraction, delaying asymptotic performance with exemplar memory.

The robustness of linear models (Dawes \& Corrigan, 1974), together with a rule bias, defines one limiting condition for the adaptation observed in this experiment: The improvement in performance by shifting from cue abstraction to exemplar memory needs to be sufficiently large to be noticed and worth the effort (Olsson, Enkvist, \& Juslin, 2006). On the other hand, at least the old-new differences suggest that the added complexity in the conditions with heterogeneous cue directions may also be a factor sufficient to increase the prevalence of exemplar memory. The results in this article suggest that the interpretation often found in the literature on multiple-cue judgment, that people mentally integrate individual cues to perform a judgment (Cooksey, 1996; Einhorn et al., 1979; Hammond \& Stewart, 2001), needs to be tested empirically as a function of task properties.

\section{AUTHOR NOTE}

This research was supported by the Bank of Sweden Tercentenary Foundation and the Swedish Research Council. L.K. is now at the Center for Adaptive Behavior and Cognition, Max Planck Institute for Human Development, Berlin. We thank Tommy Enqvist, Ebba Elwin, Patrik Hansson, Håkan Nilsson, and Anna-Carin Olsson for comments on previous versions of the manuscript. Some of the results reported in this article were presented at the 2004 Cognitive Science Conference in Chicago, Illinois. Correspondence concerning this article should be addressed to L. Karlsson, Max Planck Institute for Human Development, Lentzeallee 94, 14195 Berlin, Germany (e-mail: karlsson@mpib-berlin.mpg.de).

\section{REFERENCES}

ANDERSON, N. H. (1981). Foundations of information integration theory. New York: Academic Press.

Ashby, F. G., Alfonso-Reese, L. A., Turken, A. U., \& Waldron, E. M. (1998). A neuropsychological theory of multiple systems in category learning. Psychological Review, 105, 442-481.

Cooksey, R. W. (1996). Judgment analysis: Theory, methods, and applications. San Diego: Academic Press.

DAWES, R. M., \& CORRIGAN, B. (1974). Linear models in decision making. Psychological Bulletin, 81, 95-106.

DeLosh, E. L., Busemeyer, J. R., \& McDaniel, M. A. (1997). Extrapolation: The sine qua non for abstraction in function learning. Journal of Experimental Psychology: Learning, Memory, \& Cognition, 23, 968-986.

Einhorn, H. J., Kleinmuntz, D. N., \& Kleinmuntz, B. (1979). Linear regression and process-tracing models of judgment. Psychological Review, 86, 465-485.

Hammond, K. R., \& Stewart, T. R. (Eds.) (2001). The essential Brunswik: Beginnings, explications, applications. Oxford: Oxford University Press.

HofFMAn, P. J. (1960). The paramorphic representation of clinical judgment. Psychological Bulletin, 57, 116-131.

HogarTh, R. M., \& EInHORN, H. J. (1992). Order effects in belief updating: The belief-adjustment model. Cognitive Psychology, 24, 1-55.

JUSLIN, P., KARLSSON, L., \& OLSSON, H. (in press). Information integration in multiple-cue judgment: A division of labor hypothesis. Cognition.

Juslin, P., Olsson, H., \& Olsson, A.-C. (2003). Exemplar effects in 
categorization and multiple-cue judgment. Journal of Experimental Psychology: General, 132, 133-156.

Kalish, M. L., Lewandowsky, S., \& Krushke, J. K. (2004). Population of linear experts: Knowledge partitioning and function learning. Psychological Review, 111, 1072-1099.

Medin, D. L., \& Schaffer, M. M. (1978). Context theory of classification learning. Psychological Review, 85, 207-238.

Nosofsky, R. M. (1984). Choice, similarity, and the context theory of classification. Journal of Experimental Psychology: Learning, Memory, \& Cognition, 10, 104-114.
Olsson, A.-C., Enkvist, T., \& Juslin, P. (2006). Go with the flow! How to master a non-linear multiple-cue judgment task. Journal of Experimental Psychology: Learning, Memory, \& Cognition, 32, 13711384.

Olsson, A.-C., Juslin, P., \& Olsson, H. (2006). Individuals and dyads in a multiple-cue judgment task: Cognitive processes and performance. Journal of Experimental Social Psychology, 42, 40-56.

Shiffrin, R. M., \& SCHNEIDER, W. (1977). Controlled and automatic human information processing: II. Perceptual learning, automatic attending, and a general theory. Psychological Review, 84, 127-190.

\section{Mathematical Models and Best-Fitting Parameter Values}

Model predictions were obtained by fitting the cue-abstraction and exemplar models to the last 40 judgments of the training phase, with mean square error between predictions and data as the error function. These parameters were then applied to the exemplars in the test phase, implying cross-validation for training exemplars and genuine predictions for new exemplars. The models were fitted to individual data, and the best-fitting values appear below in Table A1.

\section{Cue Abstraction}

For each cue, the estimate of $c$ is adjusted according to the cue weight $\omega_{i \mathrm{~A}}(i=1 \ldots 4)$. The final estimate $\hat{c}_{\mathrm{CA}}$ is a linear additive function of the cues $C_{i}$,

$$
\hat{c}_{\mathrm{CA}}=k+\sum_{i=1}^{4} \omega_{i \mathrm{~A}} \cdot C_{i}
$$

where the intercept $k$ and $\omega_{i \mathrm{~A}}$ are free parameters. If $\omega_{1 \mathrm{~A}}=4, \omega_{2 \mathrm{~A}}=3, \omega_{3 \mathrm{~A}}=2$, and $\omega_{4 \mathrm{~A}}=1$, and $k=500+$ $0.5 \cdot\left(100-10 \cdot \Sigma \omega_{i \mathrm{~A}}\right)$, Equations 1 and A1 are identical, and the model produces perfect judgments (see Juslin et al., in press, and Juslin et al., 2003, for further discussion). We also fitted a multiplicative cue-abstraction model, Equation 2 of the article, with subjective cue weights and intercept.

\section{Exemplar Memory}

The estimate $\hat{c}_{\mathrm{E}}$ of the criterion $c$ is a weighted average of the criteria $c_{j}$ stored for the $J$ exemplars, where the similarities $S\left(p, x_{j}\right)$ are the weights:

$$
\hat{c}_{\mathrm{E}}=\frac{\sum_{j=1}^{J} S\left(p, x_{j}\right) \cdot c_{j}}{\sum_{j=1}^{J} S\left(p, x_{j}\right)} .
$$

$p$ is the probe to be judged, $x_{j}$ is exemplar $j(j=1 \ldots J)$, and $S\left(p, x_{j}\right)$ is the similiarity between probe $p$ and exemplar $x_{j}$. Equation A2 is the generalized context model (GCM; Nosofsky, 1984). The similarity $S\left(p, x_{j}\right)$ between exemplars is found by transforming the distance between them. The distance between a probe $p$ and an exemplar $j$ is

$$
d_{p j}=h\left[\sum_{m=1}^{M} w_{m}\left|x_{p m}-x_{j m}\right|\right],
$$

where $x_{p m}$ and $x_{j m}$, respectively, are the values of the probe and an exemplar on cue dimension $m$, the parameters $w_{m}$ are the attention weights associated with cue dimension $m$, and $h$ is a sensitivity parameter (changed from the usual $c$ to avoid confusion with the criterion $c$ ) that reflects overall discriminability in the psychological space. Attentional weights vary between 0 and 1 and are constrained to sum to 1 . The similarity $S\left(p, x_{j}\right)$ between a probe $p$ and an exemplar $j$ is assumed to be a nonlinearly decreasing function of their distance $\left(d_{p j}\right)$,

$$
S\left(p, x_{j}\right)=e^{-d_{p j}}
$$

\begin{tabular}{|c|c|c|c|c|c|c|c|c|c|c|c|c|c|c|c|}
\hline \multirow[b]{2}{*}{ Task } & \multicolumn{5}{|c|}{ CAM(A) } & \multicolumn{5}{|c|}{ CAM(M) } & \multicolumn{5}{|c|}{ EBM } \\
\hline & $\omega_{1}$ & $\omega_{2}$ & $\omega_{3}$ & $\omega_{4}$ & $k$ & $\omega_{1}$ & $\omega_{2}$ & $\omega_{3}$ & $\omega_{4}$ & $k$ & $s_{1}$ & $s_{2}$ & $s_{3}$ & $s_{4}$ & $h$ \\
\hline Additive & 3.04 & 2.22 & 1.60 & 0.74 & 511.2 & - & - & - & - & - & .28 & .29 & .24 & .19 & 3.4 \\
\hline Multiplicative & 1.41 & 0.68 & 1.07 & 0.94 & 502.3 & 2.51 & 2.01 & 2.00 & 1.90 & 514.5 & .37 & .17 & .20 & .26 & 3.5 \\
\hline
\end{tabular}

Table A1

Mean Best-Fitting Parameters for the Models in the Experiment

(Manuscript received January 15, 2007;

revision accepted for publication April 4, 2007.) 